\title{
Implicit-statistical learning in aphasia and its relation to lesion location
}

\author{
Veronika Vadinova $^{\mathrm{a}, *}, 1$, Olga Buivolova ${ }^{\mathrm{b}}$, Olga Dragoy ${ }^{\mathrm{b}, \mathrm{c}}$, Merel van Witteloostuijn ${ }^{\mathrm{a}}$, \\ Laura S. Bos ${ }^{\text {a,d }}$ \\ a University of Amsterdam, Postbus 1605, 1000 BP, Amsterdam, the Netherlands \\ ${ }^{\mathrm{b}}$ HSE University, Staraya Basmannaya st. 21/4, office 510, 105066, Moscow, Russia \\ ${ }^{\mathrm{c}}$ Federal Center for Cerebrovascular Pathology and Stroke, Ostrovityanova st. 1, 117997, Moscow, Russia \\ ${ }^{\mathrm{d}}$ Erasmus MC, Dr. Molewaterplein 40, 3015 GD Rotterdam, The Netherlands
}

\section{A R T I C L E I N F O}

\section{Keywords:}

Statistical learning

Implicit learning

Aphasia

Stroke

Syntax

Grammar

\begin{abstract}
A B S T R A C T
Background: Implicit-statistical learning (ISL) research investigates whether domain-general mechanisms are recruited in the linguistic processes that require manipulation of patterned regularities (e.g. syntax). Aphasia is a language disorder caused by focal brain damage in the left fronto-temporal-parietal network. Research shows that people with aphasia (PWA) with frontal lobe lesions manifest convergent deficits in syntax and ISL mechanisms. So far, ISL mechanisms in PWA with temporal or parietal lobe lesions have not been systematically investigated.

Aims: We investigated two complementary hypotheses: 1) the anatomical hypothesis, that PWA with frontal lesions display more severely impaired ISL abilities than PWA with posterior lesions and 2) the behavioural hypothesis, that the magnitude of impairment in ISL mechanisms correlates to syntactic deficits in aphasia. Methods: We tested 13 PWA, 5 with frontal lesions and 8 with posterior lesions, and 11 non-brain-damaged controls on a visual statistical learning (VSL) task. In addition, all PWA completed several linguistic tasks. Reaction times, obtained in the VSL task, were analyzed using linear mixed-effects model. Correlational statistics were used to assess the relationship between VSL task performance and linguistic measures.

Results and Discussion: We did not find support for the anatomical hypothesis as patients with spared frontal regions also manifested impaired ISL mechanisms. This is attributed to a) ISL mechanisms being vulnerable to other cognitive dysfunctions and/or b) ISL mechanisms anatomically extending to the posterior brain regions. Notably, ISL mechanisms were impaired, but not absent in aphasia. With regards to the behavioural hypothesis, we provide empirical evidence of correlation between ISL mechanisms and syntactic, but not lexical impairment in aphasia. We discuss both the theoretical contributions to the debate of domain-independence of ISL mechanisms and clinical implications for implicit language therapy.
\end{abstract}

\section{Introduction}

Detection, encoding and exploitation of regularities in a given environment is essential for the successful learning and use of many skills (Conway and Pisoni, 2008). Language is a prominent example of such a skill (Christiansen, 2018; Siegelman et al., 2017). Language is comprised of units at multiple levels (e.g. phonemes, syllables, words, phrases); these basic building blocks of language are assembled into complex structures, which in turn can be defined in statistical and probabilistic terms (Conway and Pissoni, 2008).

Two research traditions, Implicit learning (IL) and Statistical learning
(SL), investigate how the domain-general capacity to process regularities contributes to language acquisition and language processing (e.g. Erickson and Thiessen, 2015; Ettlinger et al., 2015; Dehaene and Cohen, 2007; Kepinska et al., 2017; van Witteloostuijn et al., 2019a). Recently, it was proposed that the two traditions be referred to under the term Implicit-statistical learning (ISL; Christiansen, 2018). It is believed that domain-general ISL mechanisms are recruited in linguistic processes that require manipulation of patterned regularities, the most prominent example being syntactic processing (Udden et al., 2017). Behavioral studies have shown that performance on ISL tasks and syntactic processing are related in healthy adults (Conway et al., 2010; Daltrozzo

\footnotetext{
* Corresponding author.

E-mail address: veronika.vadinov@gmail.com (V. Vadinova).

1 Permanent address: University of Queensland, Centre for Clinical Research, Building 71/918 RBWH Herston, Brisbane City QLD 4029, Australia.
} 
et al., 2017; Misyak and Christiansen, 2012). In the same vein, neuroimaging research has yielded robust evidence to suggest that ISL mechanisms activate anatomical regions which overlap with those activated by syntactic processing, specifically in the left inferior frontal gyrus (LIFG) in the frontal lobe (for general reviews see: Bapi et al., 2005; Conway and Pisoni, 2008; Udden and Bahlmann, 2012, and for experimental studies see: Karuza et al., 2013; Seger et al., 2000; Udden et al., 2017).

Given this behavioral and anatomical link between syntactic processing and ISL mechanisms, it has become a matter of empirical interest to study these mechanisms in people with language disorders. Aphasia is an overarching term for a series of acquired language disorders that result from brain damage in key anatomical regions or networks, most commonly after the occurrence of a stroke (Caplan, 2015). These key anatomical regions are usually dispersed across the frontal, the temporal, and the parietal lobes of the left hemisphere (Ibid.). The exact location of lesions in aphasia patients has been shown to be associated with their linguistic profiles (see Caplan, 2015 or Goodglass and Kaplan, 1994 for reviews). A particularly interesting group for analysis of ISL mechanisms is patients with Broca's aphasia, a language disorder that is characterized by deficits in complex syntactic processing (see e.g. Caplan et al., 1985; Friederici and Kotz, 2003; Novick et al., 2005). Patients with Broca's aphasia typically suffered damage to the frontal lobe, more specifically to the LIFG (Fitch and Friederici, 2012). These are the same anatomical regions that are activated during ISL tasks. This anatomical convergence has motivated a wealth of research that seeks to examine whether the syntactic impairment in Broca's aphasia might stem from a deficit in domain-general ISL mechanisms, rather than from a selective impairment to the syntax module (Cope et al., 2017; Dominey et al., 2003; Goschke et al., 2001; Schuchard and Thompson, 2013; Schuchard et al., 2016; Zimmerer et al., 2014). The majority of the aforementioned research has shown that patients with Broca's aphasia manifest impaired ISL abilities (but see Cope et al., 2017), which was suggested to support the hypothesis that ISL mechanisms play a prominent role in syntactic processing.

One limitation shared by most of these studies is that they lack an aphasic group with intact frontal regions. A second limitation is the lack of direct empirical evidence that syntactic processing and impairment in ISL mechanisms in aphasia correlate (with the exception of Dominey et al., 2003, however this study is only available as a short research summary and full study details are not provided). We aim to evaluate the performance of two anatomically defined groups of Persons With Aphasia (PWA), one with lesions in frontal regions (f-PWA) and the second with lesions in posterior regions (p-PWA), on a visual statistical learning (VSL) task in order to determine whether a lesion in the LIFG region is a prerequisite for impaired ISL abilities in aphasia. Furthermore, we aim to examine the relationship between ISL and syntactic processing in aphasia. In the subsequent paragraphs we succinctly

Box 1

Implicit learning and Statistical learning: summary

\section{Implicit learning (IL)}

IL is traditionally investigated through Artificial Grammar Learning (AGL) tasks. AGL tasks are believed to mirror natural language syntax, most often investigating relations between different types of non-adjacent dependencies (Reber, 1967; Christiansen et al., 2010; Silva et al., 2018). Example a) shows the simplest type of non-adjacent dependency in which the participant must learn that $\mathrm{A}$ and $\mathrm{B}$ are dependent, irrespective of the intervening element X. AGL grammars represent various degrees of complexity (for a review see Fitch and Friederici, 2012).

a) Non-adjacent dependency: AXB

Underlying mechanisms in IL and SL

As illustrated with the examples of both IL and SL paradigms, they study distinct structured patterns. IL focuses on the rule-based knowledge that is acquired in an AGL task. No rule-based learning is postulated to occur in an SL task, where the participant solely must gain sensitivity to the TP's of each element. These theoretical assumptions were thought to reflect the differences between syntax acquisition and lexicon learning (Perruchet and Pacton, 2006). Notwithstanding, experimental evidence from recent years showed that the two traditions may investigate overlapping functions as it is under investigation whether rule-based learning in AGL tasks also requires sensitivity to TP's of elements (as the one required for SL tasks). Furthermore, both traditions have implemented new features from each other (e.g. traditional SL tasks can now examine finite state rule-based grammars, Saffran and Wilson, 2003), and therefore a clear separation between SL and IL is no longer feasible. For a detailed review on the issue of rule-based learning vs. transitional learning, see Hauser et al. (2012); Perruchet and Pacton (2006); Silva et al. (2018). 
review the theoretically pertinent literature on ISL and the relevant findings regarding the link between language processing and ISL tasks. This is followed by a review of previous studies on ISL in aphasia.

\subsection{Implicit-statistical learning}

A marked goal of ISL research is to investigate "the human ability to detect and exploit the relations between elements in close temporal or spatial proximity" (Perruchet and Pacton, 2006, p. 237). The difference between IL and SL lies in the probed structures: while IL typically probes learning of rule-based, long-distance relationships between elements ( $i$. $e$. non-adjacent dependencies), SL usually assesses the learning of boundaries in sequential elements (i.e. adjacent dependencies). Examples of these dependencies and specific tasks associated therewith are given in Box 1. Recent theoretical reviews concluded that SL and IL share conceptual assumptions (Christiansen, 2018; Milne et al., 2018; Perruchet and Pacton, 2006), likely investigating at least partially overlapping cognitive functions and engaging partially overlapping brain regions (Karuza et al., 2013; Silva et al., 2018). In this study, we espouse the recent proposals by Milna et al. (2018) and Christiansen (2018), which state that it is empirically and experimentally beneficial not to treat these two traditions separately and will use the umbrella term ISL.

\subsection{Implicit-statistical learning and language processing: behavioral evidence}

Because language is replete with both adjacent and non-adjacent patterns, it has been assumed that domain-general ISL mechanisms play an important role in language acquisition and language processing (see e.g. Kidd et al., 2018; Siegelman et al., 2017). Several studies directly examined performance on ISL tasks and language processing $(e$. g. Conway et al., 2010; Daltrozzo et al., 2017; Misyak and Christiansen, 2012). While these studies used different ISL paradigms and compared distinct linguistic measures, all aforementioned studies found correlations between ISL mechanisms and adult language processing measures. Conway et al. (2010) found that performance on an ISL task correlated to the word-predictability measure in their study. Misyak and Christiansen (2012) used a visual AGL task with both adjacent and non-adjacent dependencies and found correlations with several syntactic measures (e.g. subject-object relative clauses, animate/inanimate relative clauses) and a word-predictability measure. Lastly, Daltrozzo et al. (2017) investigated how visual SL task performance related to grammatical and receptive vocabulary measures in an event-related potential (ERP) experiment. The authors found correlations between the ERP indices in a visual ISL task and the linguistic measure of grammatical ability. The discussed studies provide behavioral evidence of the link between visual ISL tasks and linguistic measures of syntax and word-predictability, and also show that at least partially overlapping domain-general mechanisms are utilized in both. Having explicated the behavioral link between ISL mechanisms and language processing, we proceed to elucidate the same link at the anatomical level.

\subsection{Implicit-statistical learning and language processing: anatomical evidence}

Anatomically, the LIFG corresponds to the pars opercularis, the pars triangularis, and the pars orbitalis of the left frontal lobe (cytoarchitectonically, Brodmann's areas B44, B45, and B47). A wealth of research has implicated this region as essential for syntactic processing (see Hagoort, 2005 for a review). This was corroborated by a robust finding from fMRI studies which demonstrated that higher syntactic complexity, as measured by number of clauses or number of moved elements, leads to an increase in activation of this region (e.g. Ben-Shachar et al., 2003; Friederici et al., 2005). Two opposing research traditions proposed specific models and theories to account for this finding. The first research tradition encompasses domain-specific linguistic models which postulate that the LIFG (or its subregions) is functionally specialized for syntactic operations (Grodzinsky, 2000; Friederici, 2002). A prominent model, proposed by Friederici and colleagues, postulates that left frontal regions have historically hosted general computational mechanisms for non-hierarchical (local) processing; however, a part of the left frontal regions has specialized, and now two phylogenetically younger sub-regions of the LIFG (BA44/45) are exclusively dedicated to processing the type of recursive hierarchical dependencies found in natural language syntax, namely Phrase-structure grammar (Friederici et al., 2006). In opposition to the syntax-based models, a second research tradition proposed that the LIFG region is not functionally specialized for syntactic operations, but that it rather hosts domain-general functions essential for successful syntactic processing (see Petersson et al., 2012). Within this class of models are those that argue in favor of the hypothesis that ISL mechanisms are located in the LIFG region (see Udden and Bahlmann, 2012 for a review). These models predicted a joint activation during ISL tasks (that probe the detection and the encoding of patterned regularities) and syntactic processing (that probes the exploitation of patterned regularities). Expectedly, the literature reports robust findings that processing of non-linguistic patterned regularities, such as investigated by ISL tasks, activate the same areas as syntactic processing, namely the LIFG region (e.g. AGL tasks: Petersson et al., 2012; Seger et al., 2000; Udden et al., 2017, SL boundary detection task: Karuza et al., 2013).

It is important to note that for the purpose of this study we only introduce a simplified and not fully adequate model of syntactic processing. In recent years, empirical research highlighted the importance of large language networks rather than isolated regions (see Fedorenko and Thompson-Schill, 2014). Furthermore, the notion that individual steps of language processing (e.g. lexical-semantic processing, syntactic processing) are computed in different, non-overlapping brain regions is not uncontroversial (see Blank et al., 2016 for a nuanced discussion).

\subsection{Implicit-statistical learning in aphasia}

Given the anatomical rationale of ISL research in this area, we briefly review how the lesion locations connect to the linguistic profiles of PWA. We emphasize that, while lesion location in aphasia and the linguistic profiles have been shown to be associated, caution must be exercised when drawing tentative conclusions, as this association is not always fully transparent, and there remains a large variation in linguistic deficitsdeficits (Caplan, 2015; Dronkers et al., 2004; Varkanitsa and Kasselimis, 2015). Lesions in the left frontal lobe are most commonly associated with Broca's aphasia. The strongly attested and repeatedly encountered finding is that lesions in the LIFG lead to impairment (from mild to severe) in complex syntactic processing (see e.g. Novick et al., 2005; Caplan et al., 1985; Friederici and Kotz, 2003). Not unlike language processing models, some models in aphasiology posit that the syntactic deficits in Broca's aphasia originate in a selective syntactic impairment (Grodzinsky, 2000; Friederici, 2002) while others propose deficits in domain-general cognitive mechanisms, such as resource capacity (Caplan, 2006), verbal working memory (Baldo and Dronkers, 2006), and, notably, disturbance of ISL mechanisms (e.g. Christiansen et al., 2010; Zimmerer et al., 2014). Lesions in the temporal and parietal lobes, often resulting in Wernicke's aphasia, lead to more pronounced deficits in auditory comprehension and lexical-semantic processing (see e.g. Robson et al., 2012; Thompson et al., 2015). Importantly, lesions in this area are not typically associated with the classical profile of syntactic deficits in Broca's aphasia. Patients with a lesion in these posterior areas often present severe comprehension problems, believed to result from deficits in phonological, lexical and semantic processing (see e.g. Thomson et al., 2015 for a review). Aphasia assessment batteries strive to capture the different linguistic profiles of PWA, and consequently include separate linguistic subcomponents (e.g. lexical, semantic, phonological, syntactic, non-word repetition). 
There is a dearth of studies on ISL mechanisms in aphasia (Schuchard et al., 2016). All extant studies to date have tested patients with Broca's aphasia with medium to severe syntactic impairment (Christiansen et al., 2010; Cope et al., 2017; Dominey et al., 2003), with the exception of one that also included patients without syntactic impairment (Zimmerer et al., 2014). There are pertinent differences across these studies that should be highlighted. All studies with the exception of two (Cope et al., 2017; Schuchard et al., 2016) tested a small number of patients, rendering the findings less generalizable. Dominey et al. (2003) and Christiansen et al. (2010) each administered an AGL task in agrammatic aphasia. The researchers found that the patients performed significantly worse than control participants. As a consequence, these authors assert that the syntactic deficits manifested in Broca's aphasia reflect damage to domain-general ISL mechanisms. Notably, Dominey et al. (2003) reported strong correlation between abstract sequence processing and syntactic comprehension in 7 agrammatic PWA. Unfortunately, this result is presented as a brief research summary and methodological details of this study are not available. Zimmerer et al. (2014) compared the performance of PWA with grammatical impairment to PWA without grammatical impairment on an AGL task and found that grammatical impairment was associated with a more pronounced deficit in ISL mechanisms. Zimmerer et al. (2014) did not focus on the proportion of correct vs incorrect responses, but on specific patterns of performance and strategies used by PWA as compared to controls. Considering the complex nature of the artificial grammar used in Zimmerer et al. (2014), the authors were able to analyze which types of violations were or were not rejected by PWA. While both PWA groups were impaired on the AGL task $(66 \%$ in agrammatic speakers, $70 \%$ in the aphasic group without syntactic impairment) compared to healthy participants (88\%), the authors found that the individual pattern of performance was within normal range in healthy controls and PWA without syntactic impairment. These results contradict those of Cope et al. (2017) who administered a non-word and a tone AGL task to both patients with Broca's aphasia and nonfluent Primary Progressive Aphasia (nfvPPA) and discovered that patients of both etiologies exhibited impairment as compared to controls. Nonetheless, ISL capacities were not completely absent in these groups. Lastly, Schuchard and Thompson (2013) tested 10 PWA with syntactic impairment on a different experimental paradigm that investigates learning (Serial Search Task) in order to evaluate both implicit and explicit learning deficits in aphasia. It was revealed that implicit learning was impaired to a lesser degree than explicit learning and the authors suggest that the explicit awareness and the maintenance of a sequence places excessive demands on working memory, which in turn impedes successful learning process.

\subsection{Present study}

To summarize, ISL research contends that this mechanism is at least partially domain-general and is recruited in language acquisition and language processing in addition to other functions such as perception, music appreciation and motoric skills (see Arciuli, 2016 for a review). Notably, ISL mechanisms are believed to be recruited only in the language modules that necessitate detection, encoding, and exploitation of patterned regularities, such as syntactic processing, but not in other modules like lexical recall. Two veins of evidence, anatomical and behavioral, yielded empirical support for this contention. Firstly, fMRI studies of non-linguistic ISL tasks (e.g. Karuza et al., 2013; Opitz and Friederici, 2004) found overlapping neural activation with the regions that were previously shown to activate during syntactic processing tasks, namely the LIFG region of the left hemisphere (e.g. Ben-Shachar et al., 2003; Friederici et al., 2005), giving rise to our anatomical hypothesis, which states that syntactic processing and ISL mechanisms anatomically overlap. Secondly, performance on ISL tasks and syntactic processing in adults showed a correlation (e.g. Conway et al., 2010; Daltrozzo et al., 2017), giving rise to our behavioral hypothesis, which claims that syntactic processing at least partially recruits domain-general ISL mechanisms. These two hypotheses are not competing and could be incorporated within a single linguistic model which posits that a) syntactic processing recruits ISL mechanisms and b) this mechanism is located in the LIFG region. Nonetheless, we consider it appropriate to evaluate these two hypotheses separately, as the behavioural hypothesis does not necessitate anatomical overlap, but merely argues that domain-general mechanisms are recruited in syntactic processing, irrespective of their anatomical location.

Considering the limitations in previous research, we will examine ISL abilities in two anatomically defined groups, PWA with frontal lesions (f-PWA) and PWA with posterior lesions (p-PWA), on a non-linguistic visual statistical learning (VSL) task with an additional online RT based measure. Our motivating rationale for anatomically defined groups is the prominent role that the LIFG region is assigned in ISL literature. Therefore, it was of particular interest to evaluate ISL abilities in PWA with an intact LIFG region. Furthermore, we will compare the performance on this VSL task to each patient's linguistic impairment in syntactic and lexical processing, as measured by an aphasia assessment battery. This will give us insight into the following research questions:

1. Is damage to the LIFG region a prerequisite for impaired statistical learning capacities?

2. Do syntactic processing and visual ISL mechanisms in aphasia recruit partially overlapping mechanisms?

Our first research question addresses the spared or disrupted ISL mechanisms in PWA. Based on neuroimaging evidence, we operate under the hypothesis that ISL mechanisms are grounded in the LIFG region. Therefore, we expect that f-PWA (lesioned LIFG) would display worse performance on a VSL task than p-PWA (intact LIFG) and nonbrain damaged control participants.

The second research question examines the extent to which ISL mechanisms are recruited in syntactic processing. We hypothesize, based on previous findings in aphasiology and ISL research, that the degree of syntactic impairment, as measured by aphasia assessment tasks, will correlate to the learning effect on a VSL task. The anatomical group, f-PWA or p-PWA, does not influence our predictions. F-PWA are expected to have lower scores on both the VSL task and syntactic tasks, while p-PWA are predicted to have higher scores on these two types of tasks. As a result, the anatomical group is not expected to interact with the correlational analysis. This study has not been pre-registered.

\section{Methods}

\subsection{Participants}

Three groups of participants were included in this study, all native speakers of Russian. The PWA were separated into two anatomical groups, five PWA with frontal lesions (f-PWA) and eight PWA with posterior lesions (p-PWA), while the third group comprised eleven nonbrain-damaged control participants.

PWA ( $\mathrm{N}=13$, mean age 62 years, range $50-70$ years; mean years of education 13.7 years, range 10-18 years; 7 females) were individuals with a single ischemic stroke at least two months prior to testing (mean post-onset time 13 months, range 3-46 months), all premorbidly righthanded, with no hearing or vision impairments (one PWA had corrected vision). All PWA were undergoing post-stroke rehabilitation at the Center for Speech Pathology and Neurorehabilitation (Moscow, Russia). The recruitment conditions for PWA participants were a) availability of anatomical Magnetic Resonance Images (MRI), b) brain lesion was located either in the left frontal region or left posterior (nonfrontal) region, as stated in a patient's MRI report by a local certified radiologist and confirmed with our visual inspection of native MRI images. All PWA were diagnosed with specific aphasia types and aphasia severity based on the comprehensive neuropsychological investigation by a certified clinical psychologist or a speech-and-language pathologist 
(Luria, 1964). All participants in the f-PWA group had efferent (approximately equivalent to Broca's aphasia) and afferent (comparable to conduction aphasia) motor aphasia, the linguistic profile of which is characterized by non-fluent and agrammatic speech as well as difficulties in comprehension of complex syntactic structures. Five participants in the p-PWA group were diagnosed with sensory aphasia. Their linguistic profile was characterized by a pronounced deficit in comprehension at the lexical and sentence levels, as well as paraphasias in production. One patient in the p-PWA group was diagnosed with acoustic-mnestic aphasia: a deficit in verbal working memory, with high incidence of anomia. One patient had both sensory and acoustic-mnestic aphasias; and one - efferent and afferent motor aphasias. ${ }^{2}$ PWA demographics are summarized in Appendix A, Table A.1. Lastly, PWA varied with respect to the length of the time post-stroke (3-46 months). While spontaneous and therapy-induced changes in aphasia severity can occur at any time post-stroke (Holland et al., 2016), our study focused on a single point measurement of two functions (linguistic and cognitive) that were assessed within 3 weeks of each other, which minimizes the negative influence of differences in time post-stroke. In addition, we excluded patients in the very early subacute period post-stroke (before two months) in order to assure the stability of the lesion (Heiss, 2011). Control participants $(\mathrm{N}=11$, mean age 50.2 , mean years of education 15 years, right-handed and 2 ambidextrous) presented no history of neurological and psychiatric disorders or uncorrected visual or hearing impairments.

\subsection{Materials}

\subsubsection{VSL task}

The VSL task used in the present study was developed by van Witteloostuijn et al. (2019a) to evaluate statistical learning abilities in children with developmental language impairment and is structurally based on the task in Siegelman et al. (2017). The abstract shapes that were originally employed in this VSL task were replaced by a set of 12 child-friendly multi-colored aliens (please consult Appendix B, Fig. B.1 for the entire set). The VSL task consists of two phases: the familiarization is an online RT-based task during which the participant is exposed to the visual stimuli (i.e. 12 aliens) and its aim is to measure the online learning effect; the post-familiarization phase consists of two tasks that measure the presence and the magnitude of the offline learning effect.

2.2.1.1. Familiarization phase. During familiarization, the aliens were presented to the participant on a computer screen one by one. Unknown to the participant, the aliens were repeatedly arranged in the same groups of three (i.e. triplets). In total, four triplets were used in this task (referred to as: ABC, DEF, GHI, JKL). ${ }^{3}$ The same triplet never repeated itself (i.e. disallowed: $A B C, A B C$ ) and the same rule applied to a pair of triplets (i.e. disallowed: $\mathrm{ABC}, \mathrm{DEF}, \mathrm{ABC}, \mathrm{DEF}$ ). The familiarization phase consisted of four blocks and each block contained twenty-four alien triplets ( $24 \times 4=96$ alien triplets, 288 individual aliens in total). During each block, the participant saw each of the four triplets six times. Apart from regular individual aliens, three stimuli per block contained a 'repeated alien', meaning that two aliens of the same type would appear after each other (i.e. AABC, DEEF). In such cases, the participants were instructed to touch the screen where the second alien was situated in order to 'scare him off'. This procedure was included to ensure participants' attention throughout the experiment. The statistical structure of

\footnotetext{
2 See Akhutina (2015) for a detailed review of Luria's neuropsychological investigation and the connection to the Western classification system of aphasia.

${ }^{3}$ This is a simplified version of the task in Siegelman et al. (2017)—designed to assess performance in healthy adult populations - which included eight triplets, rather than four. Considering the neurological impairment of PWA participants, a simplified version was deemed appropriate.
}

the task is reflected in transitional probabilities (TP's) of aliens. Thus, in case of a triplet $\mathrm{ABC}$, given the element $\mathrm{A}$, the TP to element $\mathrm{B}$ is 1 and the same holds for a further transition to element $\mathrm{C}$. The TP at a triplet boundary is low, as any of the other three triplets can follow. Therefore, while the element 2 and 3 (e.g. B and C) are always predictable, the element 1 (e.g. A) is unpredictable. The same principle holds for the other triplets. See Fig. 1 for an illustration of distinct TP's to predictable and unpredictable aliens. The familiarization phase constituted an online RT-based measure: participants determined for themselves the pace at which individual aliens were presented by pressing a button. After $200 \mathrm{~ms}$, a new alien would appear. In this manner, the online sensitivity to the TP's of aliens was reflected in the RT's duration. In case of successful learning, the measured RT's to predictable aliens would be shorter than RT's to unpredictable aliens.

2.2.1.2. Post-familiarization phase. Once the familiarization phase had concluded, the participant completed the post-familiarization phase, which contained two separate sub-tasks: pattern recognition alternative forced choice task (2-AFC) and pattern completion alternative force choice task (3-AFC). The tasks include grammatical, partially grammatical and ungrammatical (foil) alien triplets. Grammatical triplets appeared during the experiment, while foils were 'disallowed' alien sequences that did not appear in the familiarization phase. The 2-AFC task required that participants choose the correct pattern out of two presented alien orders; this task contained 24 questions. The 3-AFC task consisted of 16 questions, in which the participant had to fill a gap in a sequence of two or three aliens to mirror the order in which they were presented on the screen. The items in both tasks differ at two levels: number of distractors (the participant choses among 2 or 3 aliens) and difficulty (the ungrammatical pair is either partially grammatical or ungrammatical). Both 2-AFC and 3-AFC were introduced by one test trial to ensure participants understood the purpose of the task. For an example of 2-AFC and 3-AFC tasks stimuli, please consult Appendix B, Fig. 2.B.

2.2.1.3. Procedure. The VSL task was programmed and used in E-Prime 2.0 software (Schneider et al., 2002). The experiment was run on a Microsoft Surface Pro 3 with a touchscreen and a keyboard. PWA were tested in their clinical settings. Control participants were tested at various locations in Moscow, Russia.

The participants were seated in front of a tablet and told that they would engage in a short computer game. In this task, it was their mission to send lost aliens back home to their mothership. Each alien was presented individually in the middle of the screen. In order to send each alien home, participants were instructed to press the green button with the left hand. The participants were also told that, on occasion, two aliens of the same type would like to travel one after another, but that this was disallowed and, in such cases, participants were to touch the

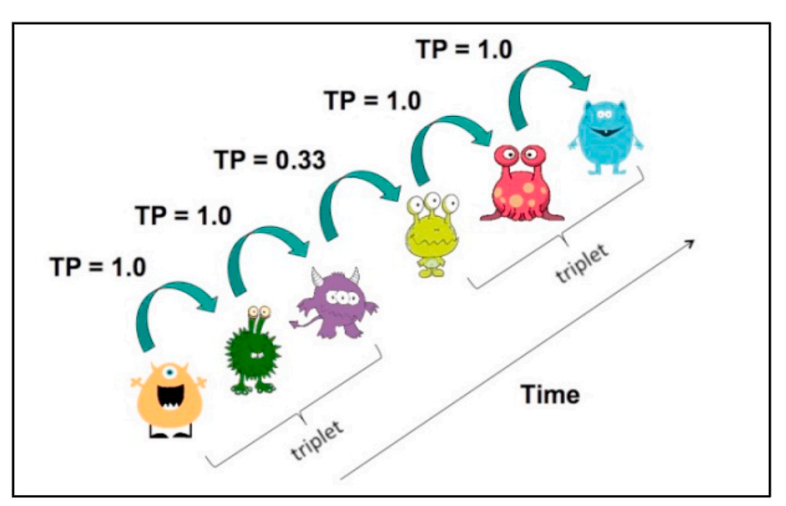

Fig. 1. Transitional probabilities of aliens within triplets (van Witteloostuijn et al., 2019a). 
screen where the alien was situated in order to scare him off. The participants were instructed to look carefully at aliens and pay attention to the order of the aliens, following the procedure in Siegelman et al. (2017) and van Witteloostuijn et al. (2019a). The familiarization phase was preceded by an instruction phase, during which the participant could practice the instructions and aims of the experiment. The instruction phase mimicked the actual familiarization phase, but did not include any of the 12 experimental aliens (i.e. additional 8 aliens were designed exclusively for the instruction phase). The familiarization consisted of four blocks, and after each block the experiment was paused and the participant could take a short break if necessary. Participants were not informed that additional post-familiarization tasks would follow. If, during the instruction phase, the experimenter believed that participants were pressing the green button inattentively or hurriedly, she instructed them to slow down and pay attention to the stimuli.

Once the familiarization phase was finished, the participant was informed that two short tasks would follow. Each task (2-AFC and 3AFC) were explained and preceded by one practice trial. In these tasks, participants had to touch the screen in order to select the correct answer. The pilot session, conducted with two PWA that could not participate in the experiment properly, revealed that PWA require a longer and more thorough instruction phase than their control counterparts. In order to ensure that all participants understood the instructions correctly, all aliens were printed and introduced to patients on paper in random order before the experiment commenced. If a patient presented persistent comprehension difficulties during the instruction phase, an additional recapitulation of the explanation was offered with the printed aliens.

\subsubsection{Linguistic tasks}

To examine our hypothesis, it was of interest to determine the syntactic and lexical deficits of PWA. Therefore, we tested PWA on two linguistic components, the syntactic measure, namely the tasks of sentence comprehension and sentence production, and the lexical measure, namely the tasks of naming and word comprehension. The comprehension and production scores were combined to derive one syntactic and one lexical measure of impairment in all PWA. Combining the production and comprehension scores was considered to provide a more comprehensive assessment of the degree of impairment in given linguistic domains (i.e. syntactic and lexical). Both tasks were taken from the Russian Aphasia Test (RAT), a standardized battery for clinical language assessment implemented on tablet (Ivanova et al., 2016). Importantly, RAT tasks that examined syntactic and lexical deficits in PWA were not used in the classification of aphasia in PWA which were decided solely on the comprehensive neuropsychological investigation (see section 2.1).

The sentence comprehension and sentence production tasks contained 24 experimental items each. These items were selected to capture varying degrees of complexity of several syntactic parameters: number of verb arguments (1-3), reversibility of the semantic roles of a verb, sentence type (simple vs. subordination), and word order (canonical vs. non-canonical). In the comprehension task, the participant had to match an auditorily presented sentence to one of the two black-and-white pictures; the production task required that the participant describes a picture with a sentence. The tasks pertaining to the lexical component were naming and word comprehension. In the naming task, the participant had to name pictures responding to the questions "What is depicted?" (for objects) and "What is a person doing in the picture?" (for actions) in one word. In the comprehension task, the participant had to match an auditorily presented word to the correct picture. The items were controlled for familiarity, visual complexity, image agreement, word imageability, age of acquisition and frequency.

The tests were administered using a Samsung Galaxy Tab A SM-T585 (2016) on Android 7.0 platform, screen size 10.1", $1920 \times 1200$ px. In the sentence comprehension test, accuracy of patients' responses was registered automatically. In the production tests, patients' vocal responses were automatically recorded by the same program and analyzed off-line later by the examiner. Linguistic tests were administered within three weeks of the VSL task.

\subsection{Data analysis}

The VSL task provided us with two outcome variables: RT's from the familiarization phase and offline accuracy scores from 2-AFC and 3-AFC tasks. These outcome variables were collected for all participants. Furthermore, linguistic data were collected for PWA which comprised two scores, syntactic and lexical impairment. Our confirmatory research questions were answered by multiple measures. As a consequence, confidence intervals were Bonferroni corrected for multiple testing, CI's were corrected to $0.05 / 4=0.0125$ (i.e. CI's $=98.75 \%$ ) for all confirmatory results. Exploratory results were not corrected (i.e. CI's 95\%).

\subsubsection{Online RT's}

Following van Witteloostuijn et al. (2019a), the RT's to the first triplet in each block were eliminated. This was done to remove deviating RT's due to the pause and restart after each block. In order to account for the variation in the individual speed of the experiment, all RT's were normalized following the exact procedure in van Witteloostuijn et al. (2019a). This was achieved by sorting all $\mathrm{N}$ observations in increasing order and replacing each observation by the $(r-0.5) / N$ quantile of the normal distribution, where $r$ is the ranking number of the observation. This normalization resulted in optimally distributed z-values. All analyses were run on normalized RT data and the model estimates are expressed as changes in z-values $(\Delta z)$ from one level of the predictor to the next (for details see van Witteloostuijn et al., 2019a). The analysis employed linear mixed-effects regression models and the lmer function of the lme4 package (Bates et al., 2015) in R (R Core Team, 2013). Once RT's were normalized, residuals became normally distributed, which rendered the application of a mixed-effects linear model suitable. All continuous variables were scaled. The online sensitivity to the transitional probabilities in the task was expressed as a difference in RT's between unpredictable (i.e. alien 1) and predictable (i.e. alien 2 and alien 3) aliens in interaction with Time (i.e. repetition of triplets across time). Predictability (categorical variable: element 1 , element 2 and element 3) and Time (continuous variable, scaled) were within-participant predictors. With regards to our first research question, the interaction with the between-participant predictor Group was of interest. Predictability and Group were contrasted using backwards difference coding. Two backwards difference codings were applied to the predictor Group. The confirmatory coding examined whether Control participants and p-PWA performed significantly different than f-PWA. The exploratory coding examined if all PWA (f-PWA and p-PWA) performed significantly worse than Control participants. A single model was thus used with two codings. Random slopes were included by-Subject and by-Item.

\subsubsection{Offline score 2-AFC and 3-AFC tasks}

Responses on both tasks were scored 0 for incorrect and 1 for correct responses. Accuracy was expressed as the percentages of correct responses. Initially we examined whether each Group displayed learning by comparing performance with chance level. This analysis was carried out separately per each task, as chance levels differed; task 2-AFC had a chance level of 0.500 and 3 -AFC of 0.333 . A one-sample $t$-test was run per task and per Group.

To answer our research question, whether the magnitude of the learning effect differed as a function of Group, we applied a glmer function from the lme4 package (Bates et al., 2015). Both tasks (2-AFC and 3-AFC) were run separately. The accuracy on the tasks was fitted as a function of Group. A single model with two codings (confirmatory and exploratory codings, see Section 2.5.1.) was used for 2-AFC and 3-AFC tasks. The statistical results from the generalized mixed-effects were exponentiated for easier interpretation (see van Witteloostuijn et al., 
2019a).

\subsubsection{Offline score and linguistic measures}

Our second research question addressed the correlation between linguistic impairment and visual statistical learning in aphasia. In order to determine whether the magnitude of the offline learning effect was related to the linguistic measures of PWA, we ran a Pearson's momentcorrelation between the 2-AFC and 3-AFC tasks and two linguistic measures: syntactic impairment and lexical impairment.

\section{Results}

In the following sections, we present results aimed at answering our two research questions. The first section focuses on visual statistical learning abilities in two aphasic anatomical groups (f-PWA and p-PWA) and Control participants. The second section addresses the relationship between visual statistical learning and linguistic measures of f-PWA and p-PWA.

\subsection{Familiarization phase: online RT's}

Our first research question examined the effect of Group on the sensitivity to the statistical regularities in the task. Fig. 2 illustrates the normalized RT's to predictable and unpredictable aliens across 24 repetitions in each Group. Mean raw and normalized RT's per Group are given in Table 1. Considering the potential Group differences, we find no evidence that p-PWA and Controls display a stronger learning effect than f-PWA ( $\Delta z=-0.031,98.75 \%$ CI $[-0.168 \ldots 0.104], t=-0.584, p=$ 0.559). In light of these results, we examined whether Controls displayed a larger learning effect than all PWA together (see Online supplementary material on details of contrast coding). This was confirmed; Control participants showed higher sensitivity to the statistical regularities of the task than PWA $(\Delta z=0.104,95 \%$ CI [0.017 ...0.192], $t=$ 2.366, $p=0.018$ ). We conclude that there is evidence of non-brain damaged participants showing a better capacity to apprehend statistical regularities than PWA.

\subsection{Post-familiarization phase: offline tasks}

Table 2 summarizes the performance on two tasks- 3-AFC (completion task) and 2-AFC (recognition task)—indicating the mean and the standard deviation per group. The mean score on the 3-AFC task (chance level $=33 \%$ ) was $64 \%$ in Control participants, $44 \%$ in f-PWA and $43 \%$ in p-PWA. The mean score on the 2 -AFC task (chance level = $50 \%$ ) was $73 \%$ in Control participants, $61 \%$ in f-PWA and $52 \%$ in p-

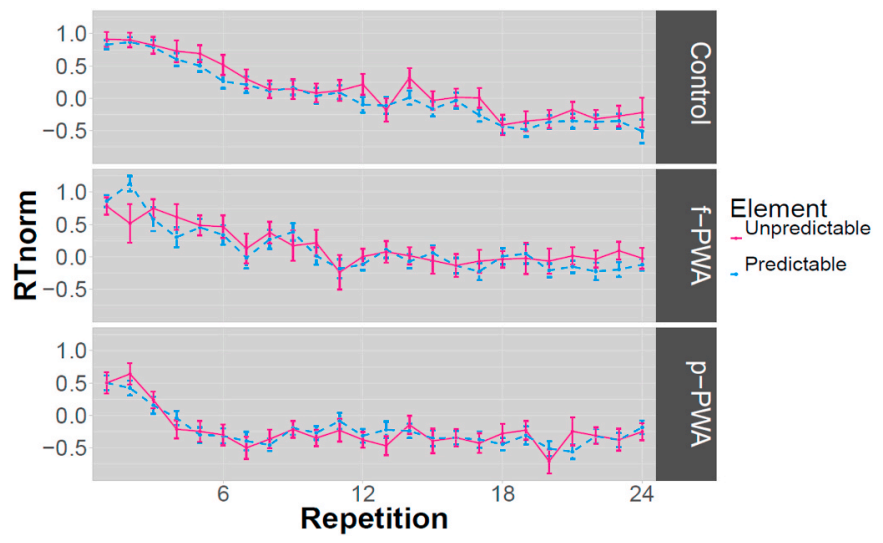

Fig. 2. VSL familiarization phase, normalized RTs ( \pm 1 SE) to predictable and unpredictable elements across repetitions of triplets (i.e. 24 repetitions) during the familiarization phase for Control participants (top graph), f-PWA (middle graph), and p-PWA (bottom graph).
Table 1

VSL familiarization phase, mean raw (ms), and normalized RT's (inside brackets) to predictable and unpredictable Aliens for three participant groups: Controls, f-PWA, and p-PWA.

\begin{tabular}{lllll}
\hline & All participants & $\begin{array}{l}\text { Controls } \\
(\mathrm{n}=11)\end{array}$ & $\begin{array}{l}\text { f-PWA } \\
(\mathrm{n}=5)\end{array}$ & $\begin{array}{l}\text { p-PWA } \\
(\mathrm{n}=8)\end{array}$ \\
\hline Predictable & $813(-.018)$ & $896(.167)$ & $837(.112)$ & $701(-0.23)$ \\
Unpredictable & $818(.036)$ & $873(.062)$ & $830(.146)$ & $715(-0.23)$ \\
\hline
\end{tabular}

Table 2

Mean scores and standard deviations in 2-AFC (chance level $=.5$ ) and 3-AFC (chance level $=.33$ ) tasks in three participant groups: Controls, f-PWA, and pPWA.

\begin{tabular}{lll}
\hline Group & 3-AFC, $\%$ & 2-AFC, $\%$ \\
\hline Control $(\mathrm{n}=11)$ & $64(9.7)$ & $73(6.9)$ \\
f-PWA $(\mathrm{n}=5)$ & $45(12.4)$ & $64(9.7)$ \\
p-PWA $(\mathrm{n}=8)$ & $43(12.6)$ & $52(10.1)$ \\
\hline
\end{tabular}

PWA.

A one sample $t$-test revealed that the performance on 2-AFC task was significantly above chance in two of the participant groups, Controls $(r$ $=0.731,98.75 \%$ CI [0.662 .. 0.799], $t=8.450, p=2.001 \mathrm{e}^{-15}$ ) and $\mathrm{f}$ PWA ( $r=0.641,98.75 \%$ CI $[0.530 \ldots 0.753], t=3.222, p=0.001)$. The performance of p-PWA was not above chance level ( $r=0.520,98.75 \%$ CI [0.429 .. 0.611], $t=0.576, p=0.565)$. Thus, we find evidence that non-brain damaged individuals and patients with frontal lesions acquired sensitivity to the structure, as measured by the 2-AFC task. Performance on the 3-AFC task was above chance level in all three groups: Control group $(r=0.642,98.75 \%$ CI $[0.550 \ldots 0.733], t=8.610, p=$ $\left.4.109 \mathrm{e}^{-15}\right)$, f-PWA $(r=0.45,98.75 \%$ CI [0.306 $\ldots 0.593], t=2.3143, p$ $=0.035)$ and p-PWA $(r=0.437,98.75 \%$ CI $[0.325 \ldots 0.549], t=2.442$, $p=0.015)$. We provide evidence that both non-brain damaged individuals and PWA learned the underlying statistical regularities as measured by the 3-AFC task.

We now introduce results with regards to Group differences on the offline tasks. Fig. 3 shows the proportion of correct responses on both tasks per Group. There is no evidence that Controls and p-PWA scored significantly higher than f-PWA on 2-AFC (log odds $=+0.582$, CI $98.75 \%=[0.121 \ldots 2.604], p=0.328)$ and 3 -AFC tasks (log odds $=$

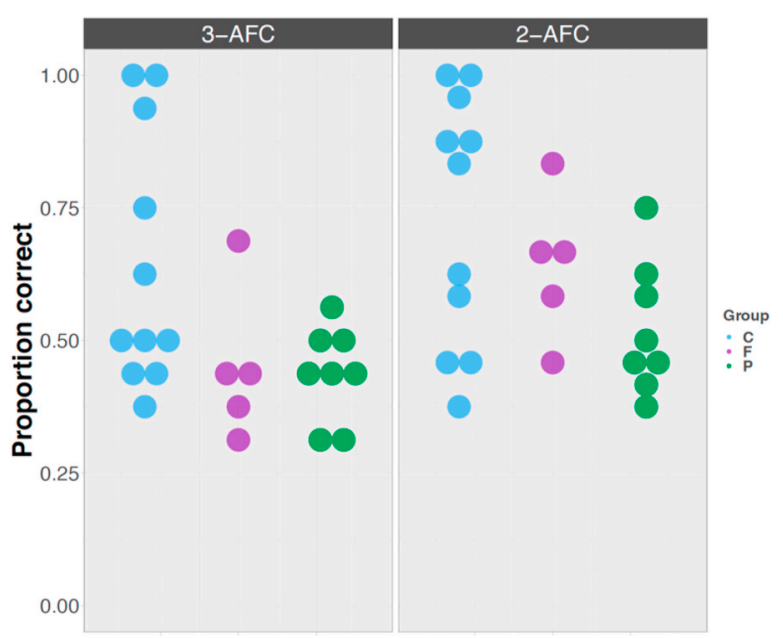

Fig. 3. VSL offline task, proportion of correct responses on 3-AFC (left, chance level $=0.33$ ) and 2-AFC questions (right, chance level $=0.5$ ) for three participant groups: Control, f-PWA, p-PWA. Color-sorted dots indicate individual scores. Controls participants (blue color), f-PWA (purple color), p-PWA (green color). (For interpretation of the references to color in this figure legend, the reader is referred to the Web version of this article.) 

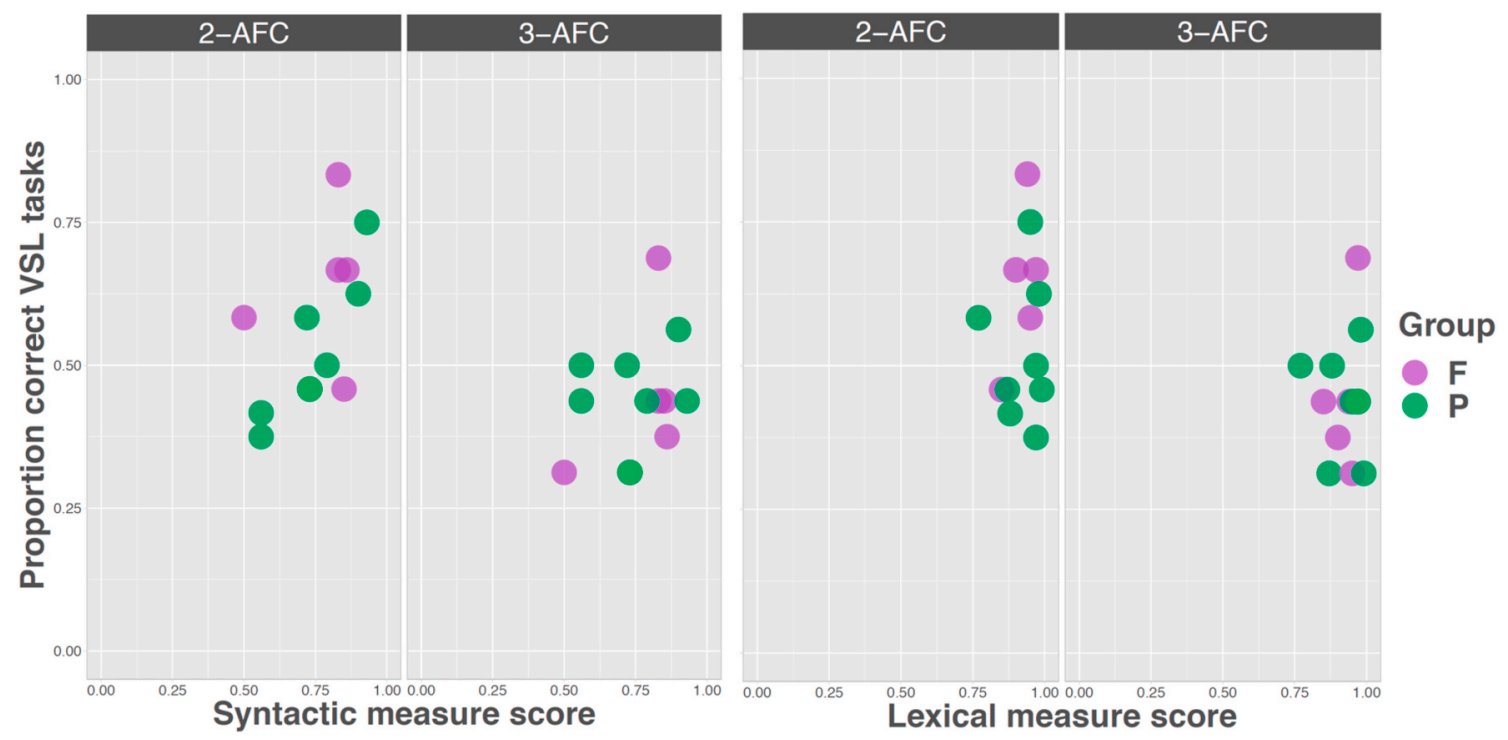

Fig. 4. Correlation of performance on linguistic measures and accuracy on 2-AFC and 3-AFC tasks. Colour-sorted dots indicate individual participants. (For interpretation of the references to color in this figure legend, the reader is referred to the Web version of this article.)

+0.582 , CI $98.75 \%=[0.270 \ldots 3.317], p=0.907)$. We subsequently examined the exploratory research question whether Controls performed significantly better than the PWA together. We provide evidence for this hypothesis on both 2-AFC and 3-AFC tasks. The Control group scored significantly higher than PWA on both the 2-AFC task (log odds $=+3.217$, CI $95 \%=[1.296 \ldots 8.775], p=0.013)$ and the 3-AFC task $(\log$ odds $=+2.536$, CI $95 \%=[1.196 \ldots 5.786], p=0.017)$. We conclude that non-brain-damaged individuals demonstrate a stronger statistical learning effect, as measured by 3-AFC and 2-AFC tasks, than PWA with frontal and posterior lesions.

\subsection{Offline tasks and linguistic measures}

Participants linguistic scores per each task are given in Appendix A. Pearson's product-moment correlation coefficients were computed to assess the correlations between the scores on VSL offline tasks 2-AFC and 3-AFC and the two linguistic measures (syntactic impairment and lexical impairment, as described in detail in Section 2.2). Fig. 4 illustrates the relationship between the linguistic scores and VSL performance on 2AFC and 3-AFC tasks. There was a weak, but significant correlation between the 2-AFC score and the syntactic impairment $(r=0.156$, CI $98.75 \%=[0.015 \ldots 0.290], p=0.005)$. No correlation was found between the 2-AFC score and the lexical impairment $(r=0.034$, CI $98.75 \%$ $=[-0.107 \ldots 0.174], p=0.542)$. Statistical results are presented in Table 3 . There was no correlation between the 3-AFC task and any of the linguistic measures (Table 3). Individual correlations between each linguistic task (naming, word comprehension, sentence production, sentence comprehension) and 2-AFC and 3-AFC tasks can be found in Appendix C, Table C.1.

\section{Discussion}

We applied a VSL task to test ISL in PWA with frontal or posterior lesions. We sought to validate two hypotheses: 1) the anatomical hypothesis: that frontal lesions lead to a more pronounced deficit in ISL mechanisms than posterior lesions in aphasia, and 2) the behavioral hypothesis: that syntactic processing and domain-general ISL mechanisms engage partially overlapping functions. Key observations regarding our first hypothesis reveal that: a) lesion location shows no relation to the magnitude of the learning effect on a VSL task (online and offline), and b) visual ISL is impaired, but not completely absent, in aphasia. The insight most pertinent to our second hypothesis shows that the syntactic deficit in PWA weakly correlated to the size of the learning effect on the VSL task. Overall, behavioral-linguistic profiles were better able to explain performance on the VSL task than general anatomical profiles. We discuss these results in turn below.

\subsection{Implicit-statistical learning in aphasia}

\subsubsection{Lesion location does not predict ISL abilities}

Our first hypothesis posited that f-PWA would manifest more severe impairment on the VSL task than p-PWA and control participants. We sought to validate the anatomical hypothesis put forward in previous research (e.g. Christiansen et al., 2010; Udden et al., 2017; Zimmerer et al., 2014): that the frontal regions-and more specifically the LIFG region-are the locus of domain-general ISL mechanisms. This hypothesis was not supported, neither in the online nor the offline tasks (Figs. 2 and 3). This result was particularly unexpected in light of the fact that all neuroimaging research strongly implied that the LIFG region plays an

Table 3

Pearson product-moment correlation between the 2-AFC and 3-AFC tasks and linguistic measures: syntactic measure score and lexical measure score. Results include size of correlation $r, 98.75 \% \mathrm{CI}$, and $p$ value.

\begin{tabular}{|c|c|c|c|c|c|c|}
\hline \multirow[t]{2}{*}{ Linguistic scores } & \multicolumn{3}{|l|}{$2-\mathrm{AFC}$} & \multicolumn{3}{|l|}{ 3- AFC } \\
\hline & $r$ & CI $98.75 \%$ & $p$ & $r$ & CI $98.75 \%$ & $p$ \\
\hline Syntactic measure score & 0.156 & {$[0.015 \ldots 0.290]$} & 0.005 & 0.049 & {$\left[\begin{array}{llll}-0.118 & \ldots & 0.213\end{array}\right]$} & 0.464 \\
\hline Lexical measure score & 0.034 & {$[-0.107 \ldots 0.174]$} & 0.542 & -0.018 & {$\left[\begin{array}{llll}-0.184 & \ldots & 0.148\end{array}\right]$} & 0.786 \\
\hline
\end{tabular}


appreciable role in the learning and processing of patterned regularities and no activation of posterior regions was reported (e.g. Karuza et al., 2013; Opitz and Friederici, 2004; Seger et al., 2000; Udden et al., 2017). There is limited research on ISL mechanisms in patients with other than Broca's aphasia. Zimmerer et al. (2014) tested PWA without syntactic impairment and concluded that their performance pattern was more similar to healthy controls. Unlike Zimmerer et al. (2014) we find no evidence of milder impairment in patients with posterior lesions when compared to patients with frontal lesions. Our results reveal that non-brained damaged individuals showed significantly better ISL than PWA, irrespective of the lesion location. Notably, our results do not invalidate previous findings that patients with lesions in frontal regions show impairment in ISL abilities. However, we provide novel evidence which suggests that PWA with spared frontal regions also exhibit impairment in ISL mechanisms. This finding adds to the current understanding of the status of ISL abilities in aphasia.

\subsubsection{Implicit-statistical learning is not absent in aphasia}

With regard to the performance of PWA, our findings suggest that ISL abilities are not totally absent in cases of aphasia. The score on the 2-AFC and 3-AFC tasks demonstrates a successful learning effect of PWA (Fig. 3). Some early studies on ISL in aphasia (Christiansen et al., 2010) have shown an absence of any learning effect on AGL tasks. Two more recent studies (Cope et al., 2017; Schuchard and Thompson, 2013; Schuchard et al., 2016), on the other hand, have evinced impaired, but not completely absent, ISL mechanism in Broca's aphasia, which is in line with the results obtained in the present study. We posit several explanations for these mixed results: a) a significant difference in sample size, and b) a difference in the structural complexity of the tasks and c) differences in socio-demographics and lesion characteristics. Firstly, all early studies tested small numbers of PWA $(n<7)$, while Cope et al. (2017) tested 22 patients with aphasia and Schuchard et al. (2016) tested 10 patients. Our data testifies to large individual variation (Fig. 3) which raises concerns regarding the validity of initial results with small samples in a heterogeneous population such as an aphasic one. Secondly, ISL tasks probe structures of varying complexity using a plethora of tasks and paradigms (see Fitch and Friederici, 2012 for a detailed discussion), which complicates cross-study comparison (Christiansen et al., 2010). We cannot reject the possibility that our finding of impaired yet present ISL abilities in patients with aphasia is due to the simpler task structure of the VSL task as compared to some AGL tasks. Due to the relatively simple underlying structure of the current VSL task, we were unable to study individual performance patterns, as is possible when investigating more complex grammars (e.g. Visser et al., 2009; Zimmerer et al., 2011, 2014). The fact that ISL mechanisms were not completely absent in PWA carries potential implications for language re-learning in aphasia. PWA with intact or mildly impaired ISL mechanisms may benefit from implicit language therapy as they are capable to detect, encode and exploit patterned regularities in the environment.

\subsection{Implicit-statistical learning and language impairment in aphasia}

\subsubsection{Implicit-statistical learning relates to linguistic measures}

ISL mechanisms are only considered to be recruited in domains that require exploitation of patterned regularities, such as syntactic processing but not lexical recall. Consequently, our working hypothesis regarding the second research question was that ISL mechanisms would correlate to the syntactic impairment, but not lexical impairment in aphasia. For this reason, we tested all PWA on two language measures - syntactic and lexical abilities-for confirmation of our hypothesis. Our findings revealed that the learning effect on the offline VSL 2-AFC task correlated with syntactic deficits in aphasia. While most ISL studies on aphasia have operated under hypotheses akin to our own- that the deficits in syntax are directly linked to the deficits in ISL abilities of patients-their empirical evidence has consisted of a convergence of deficits (except for Dominey et al., 2003, the lack of methodological details in this study are discussed in section 1.4). We consider this a key finding of our study that is in line with the direct link between syntactic processing and ISL mechanisms reported in healthy populations (e.g. Conway et al., 2010; Misyak and Christiansen, 2012). Furthermore, despite the overwhelming research focus on ISL and child language acquisition (Daltrozzo et al., 2017), we further corroborate that ISL mechanism are important to adult language processing (see Kidd et al., 2018 for a detailed discussion).

While the correlation effect between visual ISL task and syntactic deficits was significant, it was of low strength. Therefore, we only carefully suggest that our findings provide some evidence for the models that assert that language deficits in aphasia are at least partially caused by domain-general mechanisms such as ISL mechanisms, working memory, attention, or processing limitations (see Caplan, 2006 for a review).

\subsubsection{Domain-generality of implicit-statistical learning}

We want to emphasize that the magnitude of the learning effect on VSL task correlated only to syntactic impairment in aphasia and we found no evidence of a correlation between the learning effect and lexical impairment in aphasia. This finding supports the notion that, while domain-general ISL mechanisms support language processing in aphasia, they are recruited on a selective basis in language modules that require exploitation of patterned regularities. Our findings are important for debates of domain-generality of ISL functions (Frost et al., 2015; Siegelman et al., 2017). Current theoretical consensus is that ISL mechanisms operate as independent computational mechanisms but are subjected to some constraints of domains, pointing towards the coexistence of domain-general and domain-specific mechanisms (see Frost et al., 2015 for a discussion). Our data shows a direct correlation between impairment in syntax and ISL abilities. We refrain from drawing strong conclusions of complete domain-independence of ISL mechanisms, as our evidence based on two tasks is insufficient in this regard. Our findings permit us to articulate the suggestion that visual and linguistic ISL mechanisms partially overlap.

\subsection{Brain behavior relations in aphasia}

Interestingly, behavioral linguistic profiles were better able to account for the learning effect on VSL task than anatomical profiles in this study. We identified a behavioral overlap (as measured by the VSL and linguistic tests), but no anatomical overlap (as measured by the VSL and lesion location) in the present study. This is most likely caused by the heterogeneity of both size and exact location of the lesion. Our study included 13 patients, which is inadequate for a significant lesion overlap; furthermore, no matching based on the size of the lesion was performed. Stroke-induced lesions in Broca's aphasia rarely affect discrete areas, and tend to extend well beyond the language-associated Broca's area (Dronkers et al., 2004). Therefore, any conclusions regarding the localization of ISL mechanisms warrant cautious interpretation. We posit that coarse lesion information, such as in Christiansen et al. (2010), Dominey et al. (2003) or Zimmerer et al. (2014) is insufficient to draw conclusions regarding localization of ISL mechanisms. These conclusions should be relegated to fine-grained neurolinguistic methods, such as those applied in Cope et al. (2017), wherein lesion overlay with 3D atlases was used or the voxel-based lesion-symptom mapping (Bates et al., 2003).

\subsection{Limitations of the study}

Starting with the patient population, the most pertinent limitation of the present study is a small number of participants and an uneven group size. In the same vein, our study did not control for some relevant demographic variables, such as the age and the education of PWA and controls. It has been suggested that, in cases of aphasia, these variables could influence severity and recovery time (Ellis and Urban, 2016). 
Lastly, our study entails two clinical limitations: the differences in time post-stroke, and the differences in therapy stage. Unfortunately, these clinical factors were outside the scope of the current study. With regards to the first clinical limitation, the potential interaction of longitudinal aphasia changes (both positive and negative) with ISL mechanisms remains unexplored. Importantly, our study used a single time point measurement of two functions; therefore, we hypothesize that the effect of differences in time post-stroke has been minimal. Turning to the second clinical limitation, it is possible that differences in therapy stage diminished our results, as some patients have received substantially more language therapy targeting some of the relevant linguistic processes that we tested, which led to higher linguistic scores in some patients. Nonetheless, our results were significant. Future studies could include PWA that are not undergoing therapy.

Turning to the testing paradigm used, the VSL task is a popular task for measuring the capacity of individuals to apprehend statistical regularities from a given input (e.g. Daltrozzo et al., 2017; Siegelman et al., 2017). Our results show discrepancy between the online and offline findings (this was not statistically tested). Fig. 3 shows that all participants (controls and PWA) demonstrated learning on a 3-AFC task; however, no online learning effect was detected at the level of the entire group (section 3.1). We argue, in accordance with previous research (e.g. Siegelman et al., 2016), that the online component may not be sufficiently sensitive to measure participants' learning of statistical regularities in the task. This is supported by the results in van Witteloostuijn et al. (2019b), where authors failed to replicate the online learning effect identified in van Witteloostuijn et al. (2019a) in a different population. It is also plausible that the handedness procedure (all participants, PWA and controls were right-handed but instructed to complete the experiment with their left-hand) induced excessive variation in the RT's and impeded detection of the learning effect.

In addition, we cannot discard the possibility that participants in our study resorted to verbal (e.g. lexical-semantic verbalization of alien features) or non-verbal (e.g. memorization) strategies during the online familiarization phase, as we did not administer an exit questionnaire to our participants. However, descriptive findings of the exit questionnaire from the original study by van Witteloostuijn et al. (2019a) failed to demonstrate any systematic strategy applied by children when completing this task. The stimuli in this study consists of only four triplets and it could be argued that it is in the range of possibility to memorize such a small number of triplets. Importantly, during stimuli presentation the participant does not see individual triplets but only 'a sequence of triplets' (e.g. ABCGHIDEFABCJKLDEFJKLGHI). While any participant must memorize the internal structure of the triplet (e.g. $A B C$ ), this is not possible without disambiguation of the external boundary of each triplet (e.g. F-ABC-J), which we argue is accomplished via implicit-statistical learning. Nonetheless, future studies should strive to supplement the testing paradigms by exhaustive cognitive assessment of patients (e.g. verbal and non-verbal IQ, memory, processing speed, attention). It has been suggested that ISL mechanisms are of 'multi-component nature' (Arciuli, 2016) and therefore, it is plausible that unrelated cognitive dysfunctions of attention, processing speed or working memory have impacted our results.

\section{Conclusion}

In the present study we tested implicit-statistical learning in aphasia. We collected both anatomical (i.e. lesion location) and behavioural (i.e. linguistic deficits) data from all patients and compared them to the magnitude of the learning effect on a visual statistical learning task. We extend current theoretical knowledge of ISL in two ways. Our first key finding regarding the hypothesized anatomical link between ISL mechanisms and lesion location remained unsupported, and we demonstrate that patients whose LIFG regions were spared, but whose posterior regions were in fact lesioned, manifest impaired ISL mechanisms. Importantly, ISL mechanisms are not completely absent in aphasia. Our second key finding is that the magnitude of the learning effect on the VSL task weakly correlates to the impairment in syntactic abilities, but not to the impairment in lexical abilities. This is a pertinent result, as it suggests that a) domain-general ISL mechanisms at least partially overlap with mechanisms recruited in language processing and b) these ISL mechanisms are recruited in the linguistic modules that require exploitation and manipulation of patterned regularities. This was first demonstrated in typical language processing (Conway et al., 2010; Daltrozzo et al., 2017), and we validate that the same correlation holds in impaired language processing in aphasia. Turning to possible clinical implications that can be derived from our findings, it is promising that ISL mechanisms are not completely absent in aphasia. This holds potential for re-learning of damaged linguistic structures and subsequent exploitation of these structures in communication.

\section{Role of funding source}

Te study was supported within the framework of a subsidy by the Russian Academic Excellence Project '5-100'.0

\section{CRediT authorship contribution statement}

Veronika Vadinova: Conceptualization, Methodology, Investigation, Formal analysis, Writing - original draft. Olga Buivolova: Investigation, Data curation. Olga Dragoy: Conceptualization, Resources, Writing - review \& editing. Merel van Witteloostuijn: Software, Formal analysis, Writing - review \& editing. Laura S. Bos: Conceptualization, Methodology, Writing - review \& editing, Supervision.

\section{Declaration of competing interest}

None.

\section{Acknowledgements}

We are very grateful to Dirk Jan Vet for his help with the technical experiment setup and data cleaning. In addition, we would like to thank Prof J. Rispens and two further reviewers for their valuable and detailed comments on an earlier version of this paper. Lastly, we are indebted to our friend for proof-reading the first version of the manuscript, any remaining errors are our own.

\section{Appendix A. Supplementary data}

Supplementary data to this article can be found online at https://doi. org/10.1016/j.neuropsychologia.2020.107591.

\section{References}

Akhutina, T., 2015. Luria's classification of aphasias and its theoretical basis. Aphasiology 30 (8), 878-897. https://doi.org/10.1080/02687038.2015.1070950.

Arciuli, J., 2016. The multi-component nature of statistical learning. Phil. Trans. Biol. Sci. 372, 1711-1730. https://doi.org/10.1098/rstb.2016.0058.

Baldo, J.V., Dronkers, N.F., 2006. The role of inferior parietal and inferior frontal cortex in working memory. Neuropsychology 20 (5), 529-538. https://doi.org/10.1037/ 0894-4105.20.5.529.

Bapi, R.S., Pammi, V.S.C., Miyapuram, K.P., Ahmed, 2005. Investigation of sequence processing: a cognitive and computational neuroscience perspective. Curr. Sci. 89, 1690-1698.

Bates, D., Maechler, M., Bolker, B., \& Walker, S. (2015). lme4: Linear mixed-effects models using Eigen and S4 (R package version 1.1-8). Retrieved from cran.r-project. org $/$ package $=$ lme4.

Bates, E., Wilson, S.M., Saygin, A.P., Dick, F., Sereno, M.I., Knight, R.T., Dronkers, N.F., 2003. Voxel-based lesion-symptom mapping. Nat. Neurosci. 6 (5), 448-450. https:// doi.org/10.1038/nn1050.

Ben-Shachar, M., Hendler, T., Kahn, I., Ben-Bashat, D., Grodzinsky, Y., 2003. The neural reality of syntactic transformations. Psychol. Sci. 14 (5), 433-440. https://doi.org/ 10.1111/1467-9280.01459.

Blank, I., Balewski, Z., Mahowald, K., Fedorenko, E., 2016. Syntactic processing is distributed across the language system. Neuroimage 127, 307-323. https://doi.org/ 10.1016/j.neuroimage.2015.11.069. 
Caplan, D., 2006. Aphasic deficits in syntactic processing. Cortex 42 (6), 797-804. https://doi.org/10.1016/s0010-9452(08)70420-9.

Caplan, D., 2015. Aphasia. In: Wright, J.D. (Ed.), International Encyclopedia of the Social \& Behavioral Sciences, second ed., pp. 818-821. https://doi.org/10.1016/B978-008-097086-8.54022-7.

Caplan, D., Baker, C., Dehaut, F., 1985. Syntactic determinants of sentence comprehension in aphasia. Cognition 21 (2), 117-175. https://doi.org/10.1016/ 0010-0277(85)90048-4.

Christiansen, M.H., 2018. Implicit statistical learning: a tale of two literatures. Topics in Cognitive Science 3, 1-14. https://doi.org/10.1111/tops.12332.

Christiansen, M.H., Kelly, M.L., Shillcock, R.C., Greenfield, K., 2010. Impaired artificia grammar learning in agrammatism. Cognition 116, 382-393.

Conway, C.M., Pisoni, D.B., 2008. Neurocognitive basis of implicit learning of sequential structure and its relation to language processing. Ann. N. Y. Acad. Sci. 1145 (1), 113-131. https://doi.org/10.1196/annals.1416.009.

Conway, C.M., Bauernschmidt, A., Huang, S.S., Pisoni, D.B., 2010. Implicit statistical learning in language processing: word predictability is the key. Cognition 114 (3), 356-371. https://doi.org/10.1016/j.cognition.2009.10.009.

Cope, T.E., Wilson, B., Robson, H., Drinkall, R., Dean, L., Grube, M., Petkov, C.I., 2017. Artificial grammar learning in vascular and progressive non-fluent aphasias. Neuropsychologia 104, 201-213. https://doi.org/10.1016/j. neuropsychologia.2017.08.022.

Daltrozzo, J., Emerson, S.N., Deocampo, J., Singh, S., Freggens, M., Branum-Martin, L. Conway, C.M., 2017. Visual statistical learning is related to natural language ability in adults: an ERP study. Brain Lang. 166, 40-51. https://doi.org/10.1016/j bandl.2016.12.005.

Dehaene, S., Cohen, L., 2007. Cultural recycling of cortical maps. Neuron 56, 384-398. https://doi.org/10.1016/j.neuron.2007.10.004.

Dominey, P.F., Hoen, M., Blanc, J.-M., Lelekov-Boissard, T., 2003. Neurological basis of language and sequential cognition: evidence from simulation, aphasia, and ERP studies. Brain Lang. 86 (2), 207-225. https://doi.org/10.1016/S0093-934X(02) 00529-1.

Dronkers, N.F., Wilkins, D.P., Valin, R.D., Redfern, B.B., Jaeger, J.J., 2004. Lesion analysis of the brain areas involved in language comprehension. Cognition 92 (1-2), 145-177. https://doi.org/10.1016/j.cognition.2003.11.002.

Ellis, C., Urban, S., 2016. Age and aphasia: a review of presence, type, recovery and clinical outcomes. Top. Stroke Rehabil. 23 (6), 430-439. https://doi.org/10.1080/ 10749357.2016.1150412.

Erickson, L.C., Thiessen, E.D., 2015. Statistical learning of language: theory, validity, and predictions of a statistical learning account of language acquisition. Dev. Rev. 37, 66-108. https://doi.org/10.1016/j.dr.2015.05.002.

Ettlinger, M., Morgan-Short, K., Faretta-Stutenberg, M., Wong, P., 2015. The relationship between artificial and second language learning,. Cognit. Sci. 40 (4), 822-847. https://doi.org/10.1111/cogs.12257.

Fedorenko, E., Thompson-Schill, S.L., 2014. Reworking the language network. Trends Cognit. Sci. 18 (3), 120-126. https://doi.org/10.1016/j.tics.2013.12.006.

Fitch, W.T., Friederici, A.D., 2012. Artificial grammar learning meets formal language theory: an overview. Phil. Trans. Biol. Sci. 367 (1598), 1933-1955. https://doi.org/ 10.1098/rstb.2012.0103.

Friederici, A.D., 2002. Towards a neural basis of auditory sentence processing. Trends Cognit. Sci. 6 (2), 78-84. https://doi.org/10.1016/s1364-6613(00)01839-8.

Friederici, A.D., Kotz, S.A., 2003. The brain basis of syntactic processes: functional imaging and lesion studies, 20 Neuroimage. https://doi.org/10.1016/j. neuroimage.2003.09.003.

Friederici, A.D., Fiebach, C.J., Schlesewsky, M., Bornkessel, I.D., Cramon, D.Y., 2005. Processing linguistic complexity and grammaticality in the left frontal cortex. Cerebr. Cortex 16 (12), 1709-1717. https://doi.org/10.1093/cercor/bhj106.

Friederici, A.D., Bahlmann, J., Heim, S., Schubotz, R.I., Anwander, A., 2006. The brain differentiates human and non-human grammars: functional localization and structural connectivity. Proc. Natl. Acad. Sci. Unit. States Am. 103 (7), 2458-2463. https://doi.org/10.1073/pnas.0509389103.

Frost, R., Armstrong, B.C., Siegelman, N., Christiansen, M.H., 2015. Domain generality versus modality specificity: the paradox of statistical learning. Trends Cognit. Sci. 19 (3), 117-125. https://doi.org/10.1016/j.tics.2014.12.010.

Goodglass, H., Kaplan, E., 1994. The Assessment of Aphasia and Related Disorders. Lea and Febiger, Philadelphia.

Goschke, T., Friederici, A.D., Kotz, S.A., van Kampen, A., 2001. Procedural learning in Broca's aphasia: dissociation between the implicit acquisition of spatio-motor and phoneme sequences. J. Cognit. Neurosci 13 (3), 370-388.

Grodzinsky, Y., 2000. The neurology of syntax: language use without Broca's area. Behav. Brain Sci. 23, 1-71. https://doi.org/10.1017/S0140525X00002399.

Hagoort, P., 2005. On Broca, brain, and binding: a new framework. Trends Cognit. Sci. 9 (9), 416-423. https://doi.org/10.1016/j.tics.2005.07.004.

Hauser, M.F., Hofmann, J., Opitz, B., 2012. Rule and similarity in grammar: their interplay and individual differences in the brain. Neuroimage 60 (4), 2019-2026. https://doi.org/10.1016/j.neuroimage.2012.02.016.

Heiss, W.-D., 2011. The ischemic penumbra: correlates in imaging and implications for treatment of ischemic stroke. Cerebrovasc. Dis. 32 (4), 307-320. https://doi.org/ 10.1159/000330462.

Holland, A., Fromm, D., Forbes, M., Macwhinney, B., 2016. Long-term recovery in stroke accompanied by aphasia: a reconsideration. Aphasiology 31 (2), 152-165. https:// doi.org/10.1080/02687038.2016.1184221.
Ivanova, M., Dragoy, O., Akinina, J., Soloukhina, O., Iskra, E., Khudyakova, M., Akhutina, T., 2016. AutoRAT at your fingertips: introducing the new Russian Aphasia Test on a tablet. Front. Psychol. Conference Abstract: 54th Annual Academy of Aphasia Meeting. https://doi.org/10.3389/conf.fpsyg.2016.68.00116.

Karuza, E.A., Newport, E.L., Aslin, R.N., Starling, S.J., Tivarus, M.E., Bavelier, D., 2013. The neural correlates of statistical learning in a word segmentation task: an fMRI study. Brain Lang. 127 (1), 46-54. https://doi.org/10.1016/j.bandl.2012.11.007.

Kepinska, O., Rover, M.D., Caspers, J., Schiller, N.O., 2017. On neural correlates of individual differences in novel grammar learning: an fMRI study. Neuropsychologia 98 (2), 156-168. https://doi.org/10.1016/j.neuropsychologia.2016.06.014.

Kidd, E., Donnelly, S., Christiansen, M.H., 2018. Individual differences in language acquisition and processing. Trends Cognit. Sci. 22 (2), 154-169. https://doi.org/ 10.1016/j.tics.2017.11.006.

Luria, A.R., 1964. Factors and Forms of Aphasia. In: De Reuck, A.V.S., O'Connor, M. (Eds.), Disorders of Language. John Wiley \& Sons, London, pp. 143-167.

Milne, A., Wilson, B., Christiansen, M., 2018. Structured sequence learning across sensory modalities in humans and nonhuman primates. Current Opinion in Behavioral Sciences 21, 39-48. https://doi.org/10.1016/j.cobeha.2017.11.016.

Misyak, J.B., Christiansen, M.H., 2012. Statistical learning and language: an individual differences study. Lang. Learn. 62, 302-331. https://doi.org/10.1111/j.14679922.2010.00626.x.

Novick, J.M., Trueswell, J.C., Thompson-Schill, S.L., 2005. Cognitive control and parsing: reexamining the role of Broca's area in sentence comprehension. Cognit. Affect Behav. Neurosci. 5 (3), 263-281. https://doi.org/10.3758/cabn.5.3.263.

Opitz, B., Friederici, A.D., 2004. Brain correlates of language learning: the neuronal dissociation of rule-based versus similarity-based learning. J. Neurosci. 24 (39), 8436-8440. https://doi.org/10.1523/jneurosci.2220-04.2004.

Perruchet, P., Pacton, S., 2006. Implicit learning and statistical learning: one phenomenon, two approaches. Trends Cognit. Sci. 10 (5), 233-238. https://doi.org/ 10.1016/j.tics.2006.03.006.

Petersson, K., Folia, V., Hagoort, P., 2012. What artificial grammar learning reveals about the neurobiology of syntax. Brain Lang. 120 (2), 83-95. https://doi.org/ 10.1016/j.bandl.2010.08.003.

R Core Team, 2013. R: a language and environment for statistical computing. Vienna, Austria R Foundation for Statistical Computing. http://www.R-project.org/.

Reber, A.S., 1967. Implicit learning of artificial grammars. J. Verb. Learn. Verb. Behav. 6 (6), 855-863.

Robson, H., Sage, K., Ralph, M.A., 2012. Wernicke's aphasia reflects a combination of acoustic-phonological and semantic control deficits: a case-series comparison of Wernicke's aphasia, semantic dementia and semantic aphasia. Neuropsychologia 50 (2), 266-275. https://doi.org/10.1016/j.neuropsychologia.2011.11.021.

Saffran, J.R., Aslin, R.N., Newport, E.L., 1996. Statistical learning by 8-month-old infants. Science 274, 1926-1928.

Saffran, J.R., Wilson, D.P., 2003. From syllables to syntax: multilevel statistical learning by 12 -month-old infants. Infancy 4 (2), 273-284. https://doi.org/10.1207/ s15327078in0402_07.

Schneider, W., Eschman, A., Zuccolotto, A., 2002. E-prime User's Guide. Psychology Software Tools, Pittsburgh, PA.

Schuchard, J., Thompson, C.K., 2013. Implicit and explicit learning in individuals with agrammatic aphasia. J. Psycholinguist. Res 43 (3), 209-224. https://doi.org/ 10.1007/s10936-013-9248-4.

Schuchard, J., Nerantzini, M., Thompson, C.K., 2016. Implicit learning and implicit treatment outcomes in individuals with aphasia. Aphasiology 31 (1), 25-48. https:// doi.org/10.1080/02687038.2016.1147526.

Seger, C.A., Prabhakaran, V., Poldrack, R.A., Gabrieli, J.D.E., 2000. Neural activity differs between explicit and implicit learning of artificial grammar strings: an fMRI study. Psychobiology 28 (3), 283-292.

Siegelman, N., Bogaerts, L., Frost, R., 2016. Measuring individual differences in statistical learning: current pitfalls and possible solutions. Behav. Res. Methods 49 (2), 418-432. https://doi.org/10.3758/s13428-016-0719-z.

Siegelman, N., Bogaerts, L., Kronenfeld, O., Frost, R., 2017. Redefining "learning" in statistical learning: what does an online measure reveal about the assimilation of visual regularities? Cognit. Sci. 42, 692-727. https://doi.org/10.1111/cogs.12556.

Silva, S., Folia, V., Inácio, F., Castro, S.L., Petersson, K.M., 2018. Modality effects in implicit artificial grammar learning: an EEG study. Brain Res. 1687, 50-59. https:// doi.org/10.1016/j.brainres.2018.02.020.

Thompson, H.E., Robson, H., Ralph, M.A., Jefferies, E., 2015. Varieties of semantic 'access' deficit in Wernicke's aphasia and semantic aphasia. Brain 138 (12), 3776-3792. https://doi.org/10.1093/brain/awv281.

Udden, J., Bahlmann, J., 2012. A rostro-caudal gradient of structured sequence processing in the left inferior frontal gyrus. Phil. Trans. Biol. Sci. 367 (1598), 2023-2032. https://doi.org/10.1098/rstb.2012.0009.

Udden, J., Ingvar, M., Hagoort, P., Petersson, K.M., 2017. Broca's region: a causal role in implicit processing of grammars with crossed non-adjacent dependencies. Cognition 164, 188-198. https://doi.org/10.1016/j.cognition.2017.03.010.

van Witteloostuijn, M.V., Lammertink, I., Boersma, P., Wijnen, F., Rispens, J., 2019a. Assessing visual statistical learning in early-school-aged children: the usefulness of an online reaction time measure. Frontiers in Psychology 10. https://doi.org/ 10.3389/fpsyg. 2019.02051.

van Witteloostuijn, M.T.G., Boersma, P.P.G., Wijnen, F., Rispens, J.E., 2019b. Statistical learning abilities of children with dyslexia across three experimental paradigms. Plos One 14 (8), e0220041. 
Varkanitsa, M., Kasselimis, D.S., 2015. Neurological approaches to agrammatism. In: Wright, J.D. (Ed.), International Encyclopedia of the Social \& Behavioral Sciences, second ed., pp. 690-697. https://doi.org/10.1016/B978-0-08-097086-8.54022-7.

Visser, I., Raijmakers, M.E.J., Pothos, E.M., 2009. Individual strategies in artificial grammar learning. Am. J. Psychol. 122 (3), 293-307.
Zimmerer, V.C., Cowell, P.E., Varley, R.A., 2011. Individual behavior in learning of an artificial grammar. Mem. Cognit. 39, 491-501. https://doi.org/10.3758/s13421010-0039-y.

Zimmerer, V.C., Cowell, P.E., Varley, R.A., 2014. Artificial grammar learning in individuals with severe aphasia. Neuropsychologia 53, 25-38. https://doi.org/ 10.1016/j.neuropsychologia.2013.10.014. 